\title{
Exclusive photoproduction of quarkonium at the LHC energies within the color dipole approach
}

\author{
M. Beatriz Gay Ducati* \\ UFRGS, Porto Alegre \\ E-mail: beatriz.gay@ufrgs.br \\ Mirian Thurow Griep \\ UFRGS, Porto Alegre \\ E-mail: miriangriepdifsul.edu.br
}

Magno Valério Trindade Machado

UFRGS, Porto Alegre

E-mail: magnus@ufrgs.br

The exclusive photoproduction of $J / \psi$ and $\psi(2 S)$ in pp and $\mathrm{PbPb}$ collisions was investigated at the LHC energies. The theoretical framework used in the present analysis is the light-cone dipole formalism and the predictions for $J / \psi$ are compared with the ALICE and LHCb data with good agreement.

XXIII International Workshop on Deep-Inelastic Scattering

27 April - May 12015

Dallas, Texas

*Speaker. 


\section{Introduction}

The exclusive vector meson photoproduction in $\mathrm{pp}$ and $\mathrm{PbPb}$ collision allows to test perturbative Quantum Chromodynamics. The quarkonium masses, $m_{V}$, give a perturbative scale for the problem even in the photoproduction limit, $Q^{2}=0$. The Pomeron exchange can also be investigated in diffractive photoproduction of mesons at the high energy regime. At this energy domain hadrons and photons can be considered as color dipoles in the mixed light cone representation [1]. The scattering process can be characterized by the color dipole cross section representing the interaction of those color dipoles with the target (protons or nuclei). This work investigates $J / \Psi$ and $\Psi(2 S)$ states in $\mathrm{pp}$ and $\mathrm{PbPb}$ collisions at LHC energies using the dipole approach. Predictions are presented and compared with LHC data.

\section{Photon-pomeron process in relativistic nucleus-nucleus collisions}

At high energy limit and large impact parameter the nucleus-nucleus collisions are dominated by electromagnetic interaction. In this case the total cross section can be factorized in terms of the equivalent flux of photons of the hadron projectile and the photon-photon or photon-target production cross section, given by [2].

$$
\frac{d N_{\gamma}(\omega)}{d \omega}=\frac{2 Z^{2} \alpha_{e m}}{\pi \omega}\left[\xi_{R}^{A A} K_{0}\left(\xi_{R}^{A A}\right) K_{1}\left(\xi_{R}^{A A}\right) \frac{\left(\xi_{R}^{A A}\right)^{2}}{2} K_{1}^{2}\left(\xi_{R}^{A A}\right)-K_{0}^{2}\left(\xi_{R}^{A A}\right)\right],
$$

where $\omega$ is the photon energy, $\gamma_{L}$ is the Lorentz boost of a single beam and $K_{0}(\xi)$ and $K_{1}(\xi)$ are the modified Bessel functions. Considering symmetric nuclei having radius $R_{A}$, one has $\xi_{R}^{A A}=$ $2 R_{A} \omega / \gamma_{L}$.

Using the relation with the photon energy $\omega$, i.e. $y \propto \ln \left(2 \omega / m_{X}\right)$, the rapidity distribution $y$ for quarkonium photoproduction in nucleus-nucleus collisions can be written as.

$$
\frac{d \sigma[A A \rightarrow A \otimes \psi(2 S) \otimes X]}{d y}=\omega \frac{d N_{\gamma}(\omega)}{d \omega} \sigma_{\gamma A \rightarrow \psi(2 S) X}(\omega),
$$

where $\otimes$ represents the presence of a rapidity gap. Consequently, given the photon flux, the rapidity distribution is thus a direct measure of the photoproduction cross section for a given energy.

The skewedness effect (off-diagonal gluon exchange) and real part of the amplitude were considered in these predictions. The photon wavefunctions are relatively well known [3]. For the meson wave function the boosted gaussian wavefunction was considered. This simplification of the NNPZ wavefunction was presented in Refs. $[1,4]$. It has been compared to recent analysis of DESY-HERA data for vector meson exclusive processes.

The exclusive $\psi(2 S)$ photoproduction off nuclei for coherent and incoherent processes can be simply computed in high energies where the large coherence length $l_{c} \gg R_{A}$ is fairly valid. In such case the transverse size of $c \bar{c}$ dipole is frozen by Lorentz effects. The expressions for the coherent and incoherent cross sections are given by [5],

$$
\begin{aligned}
\sigma_{c o h}^{\gamma A} & =\int d^{2} b\left|\left\langle\Psi^{V}\left|1-\exp \left[-\frac{1}{2} \sigma_{d i p}(x, r) T_{A}(b)\right]\right| \Psi^{\gamma}\right\rangle\right|^{2}, \\
\sigma_{i n c}^{\gamma A} & =\frac{1}{16 \pi B_{V}(s)} \int d^{2} b T_{A}(b) \times\left|\left\langle\Psi^{V}\left|\sigma_{d i p}(x, r) \exp \left[-\frac{1}{2} \sigma_{d i p}(x, r) T_{A}(b)\right]\right| \Psi^{\gamma}\right\rangle\right|^{2} .
\end{aligned}
$$



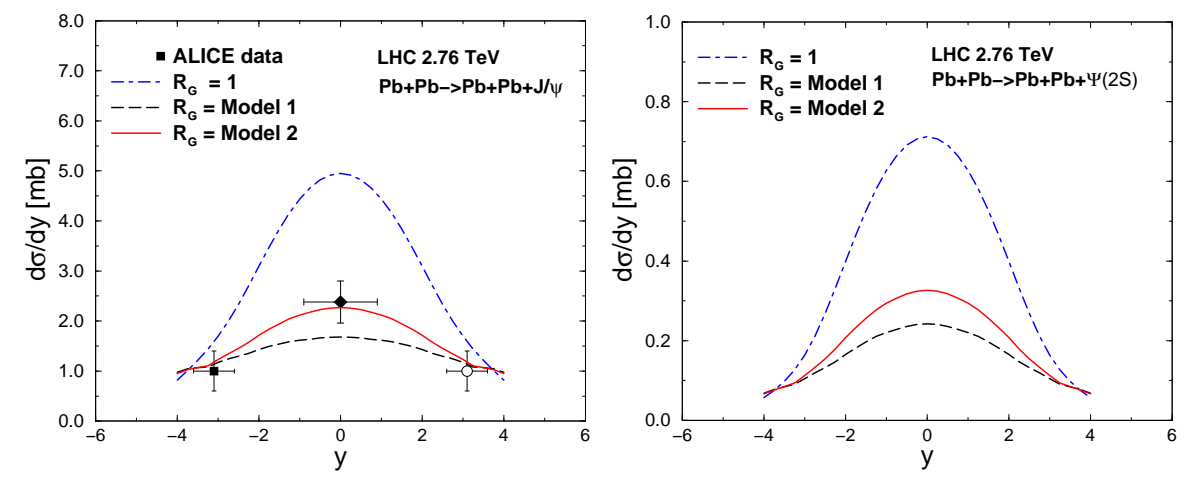

Figure 1: The rapidity distribution of coherent $\psi(1 S)$ (left) and $\psi(2 S)$ (right) meson photoproduction at $\sqrt{s}=2.76 \mathrm{TeV}$ in PbPb collisions at the LHC.

where $T_{A}(b)=\int d z \rho_{A}(b, z)$ is the nuclear thickness function given by integration of nuclear density along the trajectory at a given impact parameter $b$. In addition, $B_{V}$ is the diffractive slope parameter in the reaction $\gamma^{*} p \rightarrow \psi p$.

For the dipole cross section the Color Glass Condensate model [6] was considered for $\sigma_{d i p}(x, r)$. This model has been tested for a long period against DIS, diffractive DIS and exclusive production processes in $e p$ collisions. Corrections due to gluons shadowing were also considered as the gluon density in nuclei; at small- $x$ region is known to be suppressed compared to a free nucleon. That is, we will take $\sigma_{d i p} \rightarrow R_{G}\left(x, Q^{2}, b\right) \sigma_{d i p}$ following studies in Ref. [7]. The factor $R_{G}$ is the nuclear gluon density ratio. In the present investigation we will use the nuclear ratio from the leading twist theory of nuclear shadowing based on generalization of the Gribov-Glauber multiple scattering formalism as investigated in Ref. [8]. We used the two models available for $R_{G}\left(x, Q^{2}\right)$ in [8], Models 1 and 2, which correspond to higher nuclear shadowing and lower nuclear shadowing, respectively.

\section{Results and discussions}

The Fig. 1 (left) presents the numerical calculations for the rapidity distribution of coherent $\psi(1 S)$ state within the color dipole formalism, Eqs. (4.2) and (2.3), using distinct scenarios for the nuclear gluon shadowing [9]. The dot-dashed curve represents the result using $R_{G}=1$ and it is consistent with previous calculations using the same formalism [10]. The ALICE data is overestimate on the backward (forward) and mainly in central rapidities. The threshold factor for $x \rightarrow 1$ was not included in the present calculation, so the overestimation in the backward/forward rapidity case, is already expected. In that kinematical region either a small- $x$ photon scatters off a large- $x$ gluon or vice-versa. The ALICE data [11] is overestimate by a factor 2 considering $R_{G}=1$, as already noticed in recent study of Ref. [12]. If we consider nuclear shadowing renormalizing the dipole cross section, the situation is improved due to the gluon density in nuclei; at small Bjorken $x$ is expected to be suppressed compared to a free nucleon due to interferences. For the ratio of the gluon density, $R_{G}\left(x, Q^{2}=m_{V}^{2} / 4\right)$, we have considered the theoretical evaluation of Ref. [8]. There, two scenarios for the gluon shadowing are investigated: Model 1 corresponds to a strong gluon shadowing and Model 2 concerns small nuclear shadowing. The consequence of renormalizing the 


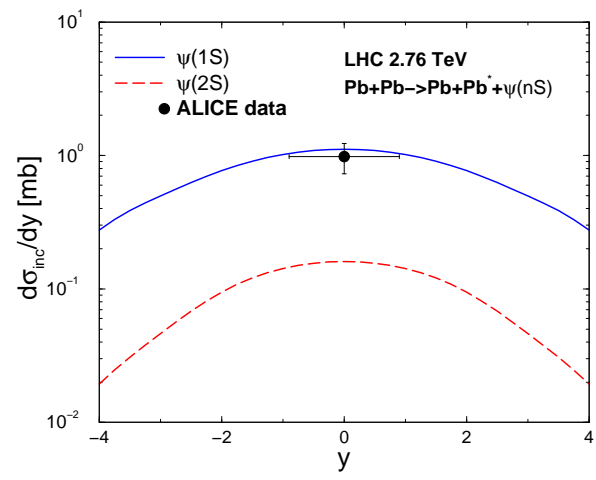

Figure 2: The rapidity distribution of incoherent $\psi(1 S)$ (solid line) and $\psi(2 S)$ (dashed line) meson photoproduction at $\sqrt{s}=2.76 \mathrm{TeV}$. Data from ALICE collaboration [11].

dipole cross section by gluon shadowing effects is represented by the long-dashed (Model 1) and solid (Model 2) lines, respectively. In the current analysis, the small shadowing option is preferred . The theoretical uncertainty related to the choice of meson wavefunction is relatively large. $R_{G}$ was considered as independent on the impact parameter.

The Fig. 1 (right) shows the first estimate in literature for the coherent photoproduction of $\psi(2 S)$ state in nucleus-nucleus collisions. In particular, for $R_{G}=1$ one obtains for central rapidity $\frac{d \sigma}{d y}(y=0)=0.71 \mathrm{mb}$ and the following in the forward/backward region $\frac{d \sigma}{d y}(y= \pm 3)=0.16$ $\mathrm{mb}$. When introducing the suppression in dipole cross section due to nuclear shadowing, one gets instead $\frac{d \sigma}{d y}(y=0)=0.24 \mathrm{mb}$ and $0.33 \mathrm{mb}$ for Model 1 and Model 2, respectively. At central rapidities, the meson state ratio is evaluated to be $R_{\psi}^{y=0}=\frac{\sigma_{\psi(2 S)}}{d y} / \frac{d \sigma_{\psi(1 S)}}{d y}(y=0)=0.14$ in case $R_{G}=1$ which is consistent with the ratio measured in CDF, i.e. $0.14 \pm 0.05$, on the observation of exclusive charmonium production at $1.96 \mathrm{TeV}$ in $p \bar{p}$ collisions [13]. A similar ratio is obtained using Model 1 and Model 2 at central rapidity as well. As a prediction for the planned LHC run in $\mathrm{PbPb}$ mode at $5.5 \mathrm{TeV}$, we obtain $\frac{d \sigma_{\text {coh }}}{d y}(y=0)=1.27 \mathrm{mb}$ and $\frac{d \sigma_{\text {inc }}}{d y}(y=0)=0.27 \mathrm{mb}$ for the coherent and incoherent $\psi(2 S)$ cross sections (upper bound using $R_{G}=1$ ), respectively.

The Fig. 2 presents the incoherent contribution for the rapidity distribution for both $\psi(1 S)$ (solid line) and $\psi(2 S)$ (dashed line) meson states[9]. For the $\psi(1 S)$ state, the present calculation can be directly compared with those studies presented in Ref. [12]. The incoherent cross section $\frac{d \sigma_{\text {inc }}}{d y}$ ranges between 0.5 to $0.7 \mathrm{mb}$ (using IIM dipole cross section) or between 0.7 to $0.9 \mathrm{mb}$ (using fIPsat dipole cross section) at central rapidities, with the uncertainty determined by the distinct meson wavefunction considered [12]. Here, was obtained $\frac{d \sigma_{\text {inc }}}{d y}(y=0)=1.1 \mathrm{mb}$ using a different expression for the incoherent amplitude, Eq. (2.4). This result describes the recent ALICE data [11] for the incoherent cross section at mid-rapidity, $\frac{d \sigma_{i n c}^{\text {ALCE }}}{d y}(-0.9<y<0.9)=0.98 \pm 0.25 \mathrm{mb}$. For the $\psi(2 S)$ state, was found $\frac{d \sigma_{\text {inc }}}{d y}=0.16 \mathrm{mb}$ for central rapidities. In both cases it was only computed the case for $R_{G}=1$. Therefore, this gives an upper bound for the incoherent cross section compared to Model 1 and Model 2 calculation. For the incoherent case, the gluon shadowing is weaker than the coherent case and the reduction is around $20 \%$ compared to the case $R_{G}=1$. The incoherent piece is quite smaller compared to the main coherent contribution. As an example of order of 

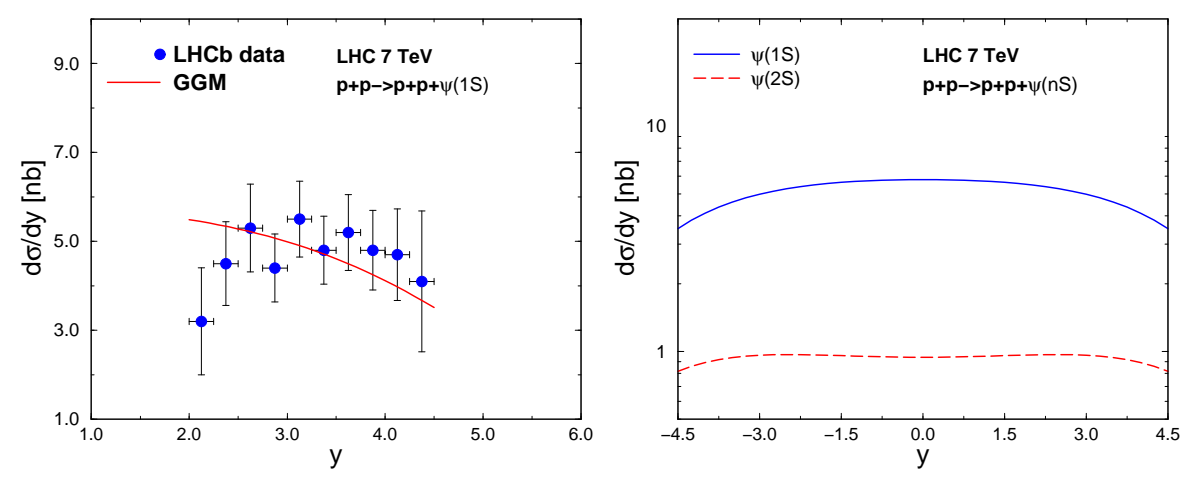

Figure 3: The rapidity distribution at forward region of exclusive $\psi(1 S)$ production (left) and rapidity distribution of exclusive $J / \psi$ (solid curve) and $\psi(2 S)$ (dashed curve) production (right) at $\sqrt{s}=7 \mathrm{TeV}$ in proton-proton collisions at the LHC.

magnitude, the ratio incoherent/coherent is a factor 0.22 for the $1 S$ state and 0.23 for the $2 S$ state at central rapidity.

\section{Exclusive meson photoproduction in proton-proton collisions}

The cross section for the exclusive meson photoproduction in hadron-hadron collisions can also be factorized in terms of the equivalent flux of photons of the hadron projectile and photontarget production cross section [2]. The photon energy spectrum, $d N_{\gamma} / d \omega$, is given by a modified version of Wiezsäcker-Williams approximation [2]

$$
\frac{d N_{\gamma}(\omega)}{d \omega}=\frac{\alpha_{e m}}{2 \pi \omega}\left[1+\left(1-\frac{2 \omega}{\sqrt{s}}\right)^{2}\right] \times\left(\ln \xi-\frac{11}{6}+\frac{3}{\xi}-\frac{3}{2 \xi^{2}}+\frac{1}{3 \xi^{3}}\right),
$$

where $\omega$ is the photon energy and $\sqrt{s}$ is the hadron-hadron centre-of-mass energy. Given the Lorentz factor of a single beam, $\gamma_{L}=\sqrt{s} /\left(2 m_{p}\right)$, one has that $\xi=1+\left(Q_{0}^{2} / Q_{\min }^{2}\right)$ with $Q_{0}^{2}=0.71$ $\mathrm{GeV}^{2}$ and $Q_{\min }^{2}=\omega^{2} / \gamma_{L}^{2}$. The rapidity distribution $y$ for charmonium photoproduction, i.e. the $\psi(1 S)$ and $\psi(2 S)$ states, in proton-proton collisions can be written down as,

$$
\frac{d \sigma}{d y}(p p \rightarrow p \otimes \psi \otimes p)=S_{g a p}^{2}\left[\omega \frac{d N_{\gamma}}{d \omega} \sigma(\gamma p \rightarrow \psi(n S)+p)+(y \rightarrow-y)\right],
$$

where $\otimes$ represents the presence of a rapidity gap. The produced state with mass $m_{V}$ has rapidity $y \simeq \ln \left(2 \omega / m_{V}\right)$ and the square of the $\gamma p$ centre-of-mass energy is given by $W_{\gamma p}^{2} \simeq 2 \omega \sqrt{s}$. The absorptive corrections due to spectator interactions between the two hadrons are represented by the factor $S_{\text {gap }}$. The corrections related to skewedness effect and real part of amplitude are also considered. For the charm quark mass, we will use the value $m_{c}=1.4 \mathrm{GeV}$. The CGC model was used for the dipole cross section [6].

\section{Results and discussions}

Fig. 3 (left) compares the present theoretical approach to the data for the $\psi(1 S)$ state measured by $\mathrm{LHCb}$ Collaboration at the energy of $7 \mathrm{TeV}$ in proton-proton collisions at the forward region 
$2.0<\eta_{ \pm}<4.5$ [14]. Hereafter, we will assume for the absorption factor the average value $S_{\text {gap }}^{2}=$ 0.8 , despite it depends on rapidity as shown in Ref. [15]. The absorptive corrections considering the elastic rescattering have been computed for $p p$ collisions in [15] and have a value of $S_{\text {gap }}^{2}(y=$ $0)=0.85$ and $S_{\text {gap }}^{2}(y=3)=0.75$, respectively. In Fig. 3 the numerical calculations (labeled GGM and represented by the solid curve) are shown for the rapidity distribution for $\psi(1 S)$ state within the color dipole formalism, Eq. (4.2). The relative normalization and overall behavior on rapidity is quite well reproduced in the forward regime. In Fig. 3 the complete rapidity distribution, including mid-rapidity and backward region, is presented for $J / \psi$ and $\psi(2 S)$ states (solid and dashed curves, respectively). The $J / \psi$ cross section at central rapidity is $\frac{d \sigma}{d y}(y=0)=5.8 \mathrm{nb}$. We obtain $\sigma(p p \rightarrow$ $p+J / \psi+p) \times \operatorname{Br}\left(J / \psi \rightarrow \mu^{+} \mu^{-}\right)=698 \mathrm{pb}$ for the meson with a rapidity between 2 and 4.5 . After correcting this result by the acceptance factor in order to convert the prediction in terms of muon pseudorapidities we get $\sigma_{p p \rightarrow J / \psi\left(\rightarrow \mu^{+} \mu^{-}\right)}\left(2.0<\eta_{\mu^{ \pm}}<4.5\right)=298 \mathrm{pb}$. This is in good agreement to the experimental result $\sigma_{p p \rightarrow J / \psi\left(\rightarrow \mu^{+} \mu^{-}\right)}\left(2.0<\eta_{\mu^{ \pm}}<4.5\right)=307 \pm 42 \mathrm{pb}$ [14] (summing errors in quadrature).

The Fig. 3 (right) shows the rapidity distribution for excited $\psi(2 S)$ mesons (dashed line) [9], which gives at central rapidity a cross section $\frac{d \sigma}{d y}(y=0)=0.94 \mathrm{nb}$. It is obtained $\sigma(p p \rightarrow$ $p+\psi(2 S)+p) \times \operatorname{Br}\left(\psi(2 S) \rightarrow \mu^{+} \mu^{-}\right)=18 \mathrm{pb}$ for rapidities $2.0<y<4.5$. Accordingly, we now predict $\sigma_{p p \rightarrow \psi(2 S)\left(\rightarrow \mu^{+} \mu^{-}\right)}\left(2.0<\eta_{\mu^{ \pm}}<4.5\right)=7.7 \mathrm{pb}$ compared to $\sigma_{p p \rightarrow \psi(2 S)\left(\rightarrow \mu^{+} \mu^{-}\right)}\left(2.0<\eta_{\mu^{ \pm}}<\right.$ $4.5)=7.8 \pm 1.6 \mathrm{pb}$ measured by $\mathrm{LHCb}[14]$. At mid-rapidity we obtain the ratio $[\psi(2 S) / \psi(1 S)]_{y=0}$ $=0.16$ and taking the integrated cross section for $2.0<y<4.5$ we have $[\psi(2 S) / \psi(1 S)]$ $2<y<4.5=0.18$. The latter value of the ratio is strongly consistent to the LHCb determination $[\psi(2 S) / \psi(1 S)]\left(2.0<\eta_{\mu^{ \pm}}<4.5\right)=0.19 \pm 0.04$.

It is timely to compare our results to the similar theoretical approaches in literature. The values obtained for the integrated cross sections for the exclusive $J / \psi$ production are consistent with calculations using the color dipole formalism [10,16] and with the prediction from Starlight [17] and SuperChic [18] generators as well. In the case of $\psi(2 S)$ state our prediction is in agreement to the Starlight generator result, which gives $\sigma_{p p \rightarrow \psi(2 S)\left(\rightarrow \mu^{+} \mu^{-}\right)}^{\mathrm{STARLIGHT}}=6.1 \mathrm{pb}$. On the other hand, our results are a factor about 2 lower than the values appearing in Ref. [15] that considers the $k_{\perp}$-factorization approach. The complexity of the phenomenological model considered here could turn out its connection to the QCD dynamics not so clear. Therefore, some comments are in order at this point. The main dependence being probed in the exclusive vector meson production in $p p$ collisions is the energy behavior of the photoproduction cross section (give that the photon flux is well known). The LHCb data cover one order of magnitude on photon-proton centre-of-mass energy above the typical DESY-HERA regime. Such an extrapolation is completely driven by the QCD dynamics at small-x and embeded in the dipole cross section in our analysis. This is directly translated into the rapidity dependence of $p p$ cross section. The other inputs like the paramaters in wavefunctions, real part and skewdness corrections and absorption effects only account for the overall normalization. It's worth mentioning that our predictions are parameter free. The inputs in the meson wavefunction are determined from its normalization condition. The phenomenological parameters in dipole cross section were determined by a fit to DESY-HERA data for proton structure function $F_{2}$ at small-x [6] and already tested against exclusive processes at DESY-HERA energies at a number of contributions $[19,16]$. 


\section{Summary}

The photoproduction of radially excited vector mesons was investigated in $\mathrm{pp}$ and $\mathrm{PbPb}$ collisions at LHC energies using the light-cone dipole formalism. Predictions are done for $\mathrm{PbPb}$ collisions at the CERN-LHC energy of $2.76 \mathrm{TeV}$. The fact that the gluons shadowing suppress the dipole cross section was studied and the results for $R_{G}=1$ give the larger cross sections. The coherent exclusive photoproduction of $\psi(2 S)$ off nuclei has an upper bound of order $0.71 \mathrm{mb}$ at $y=0$ down to $0.10 \mathrm{mb}$ for backward/forward rapidities $y= \pm 3$. The incoherent contribution was also computed and it is a factor 0.2 below the coherent one. A small nuclear shadowing $R_{G}\left(x, Q^{2}=\frac{m_{V}^{2}}{4}\right)$ is preferred in ALICE data description whereas the usual $R_{G}=1$ value overestimates the central rapidity cross section by a factor 2 for the $\psi(1 S)$ state photoproduction. For incoherent cross section, the present theoretical approach describes the ALICE data. Thus, the central rapidity data measured by ALICE Collaboration for the rapidity distribution of the $\psi(1 S)$ state is crucial to constrain the nuclear gluon function. For pp collisions it was found that the the coherent exclusive photoproduction of $\psi(2 S)$ off nuclei has an upper bound of order $0.71 \mathrm{mb}$ at $y=0$ down to 0.10 $\mathrm{mb}$ for backward/forward rapidities $y= \pm 3$. The incoherent contribution was also computed and it is a factor 0.2 below the coherent one. Comparison has been done to the recent LHCb Collaboration data for the exclusive $\psi(1 S)$ and $\psi(2 S)$ production at $7 \mathrm{TeV}$. The experimental values are fairly described by the present calculation.

\section{References}

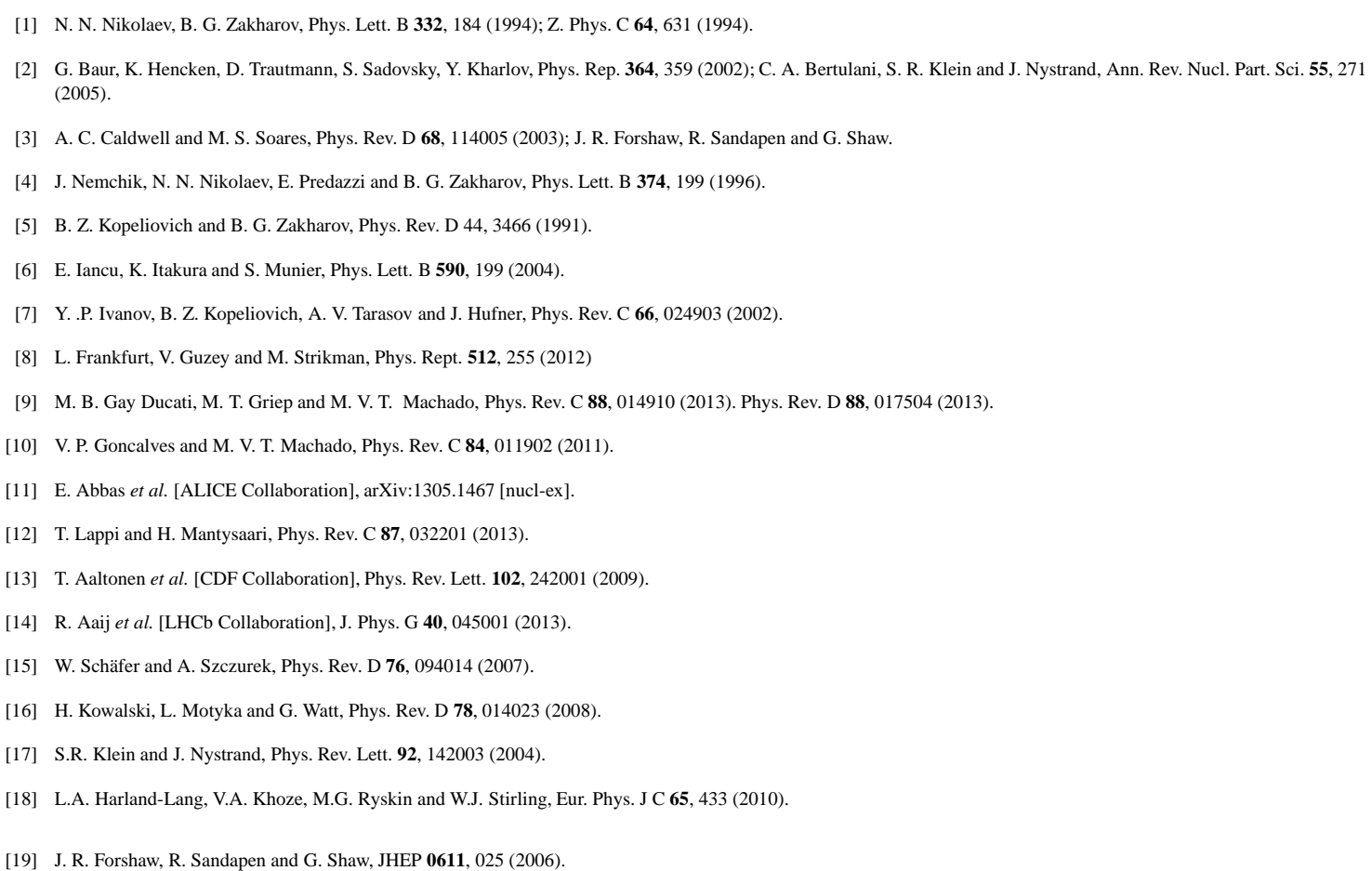

